\title{
Effects of wavelength-dependent fluence attenuation on the noninvasive photoacoustic imaging of hemoglobin oxygen saturation in subcutaneous vasculature in vivo
}

Hao F. Zhang, Konstantin Maslov, Lihong V. Wang

Hao F. Zhang, Konstantin Maslov, Lihong V. Wang, "Effects of wavelengthdependent fluence attenuation on the noninvasive photoacoustic imaging of hemoglobin oxygen saturation in subcutaneous vasculature in vivo," Proc. SPIE 6856, Photons Plus Ultrasound: Imaging and Sensing 2008: The Ninth Conference on Biomedical Thermoacoustics, Optoacoustics, and Acoustooptics, 68561T (28 February 2008); doi: 10.1117/12.761984 


\title{
Effects of wavelength-dependent fluence attenuation on the noninvasive photoacoustic imaging of hemoglobin oxygen saturation in subcutaneous
}

\section{vasculature in vivo}

\author{
Hao F. Zhang ${ }^{1}$, Konstantin Maslov ${ }^{2}$, and Lihong V. Wang ${ }^{2}$ \\ 1. Department of Electrical Engineering and Computer Science, The University of Wisconsin- \\ Milwaukee, P O Box 784, Milwaukee, WI 53201 \\ 2. Optical Imaging Laboratory, Department of Biomedical Engineering, Washington University \\ in St. Louis, Campus Box 1097, St. Louis, MO 63130
}

Correspondence should be addressed to Lihong V. Wang. (lhwang@biomed.wustl.edu).

\begin{abstract}
Due to wavelength-dependent optical attenuation in the skin, the local fluence at a subcutaneous vessel varies with the optical wavelength in a spectral measurement. Hence compensation for such a spectral attenuation is necessary in quantitative measurements of the oxygen saturation of hemoglobin $\left(\mathrm{sO}_{2}\right)$ in blood vessels in vivo using photoacoustic (PA) imaging. Here, by employing a simplified double-layer skin model, we find that although the absolute value of $\mathrm{sO}_{2}$ in a vessel is seriously affected by the volume fraction of blood and the spatially averaged $\mathrm{sO}_{2}$ in the dermis, the difference of $\mathrm{sO}_{2}$ between neighboring vessels is minimally affected. Based on in vivo experiments, we demonstrate that the difference in $\mathrm{sO}_{2}$ between a typical artery and a typical vein is conserved before and after an experimentally acquired spectral compensation. This conservation holds regardless of the animal's systemic physiological state.
\end{abstract}

Keywords Photoacoustic microscopy, hemoglobin oxygen saturation, fluence attenuation

\footnotetext{
Photons Plus Ultrasound: Imaging and Sensing 2008: The Ninth Conference on Biomedical Thermoacoustics, Optoacoustics, and Acousto-optics, edited by Alexander A. Oraevsky, Lihong V. Wang, Proc. of SPIE Vol. 6856, 68561T, (2008) · 1605-7422/08/\$18 - doi: 10.1117/12.761984
} 


\section{Introduction}

Photoacoustic (PA) imaging is based on the detection of the laser induced ultrasonic waves, which are resulted from optical absorption and thermoelastic expansion. The PA images, either reconstructed in photoacoustic tomography (PAT) [1-5] or acquired directly in photoacoustic microscopy (PAM) [6-8], describes the internal distribution of the optical energy deposition, which is the product of the local fluence and the tissues' optical absorption coefficient. Therefore, PA images are well suited to reveal optical absorption contrast but, at the same time, can be biased in depicting tissue's optical absorption coefficient distribution if there is a large variation in local fluence. Besides optical heterogeneity, local fluence also varies with optical wavelengths due to the wavelength-dependent optical absorption coefficient and reduced optical scattering coefficient in the surrounding tissues, such as skin. To minimize the variations in local fluence, one can choose a spectral range where optical attenuation in skin is low, i.e. between $650 \mathrm{~nm}$ and $850 \mathrm{~nm}$. However, within this spectral range, only a low signal-to-noise ratio can be achieved since the optical absorption of the targeted structure also decreased. Hence, quantitative imaging of functional parameters, such as hemoglobin oxygen saturation $\left(\mathrm{sO}_{2}\right)$, especially with high spatial resolution, is quite challenging.

In PA imaging of $\mathrm{sO}_{2}$ in subcutaneous vessels, spectral measurement is employed to extract the relative concentration of the oxyhemoglobin $\left(\mathrm{HbO}_{2}\right)$ and deoxyhemoglobin $(\mathrm{HbR})$ based on their distinctive optical distinction coefficient spectra. Therefore compensating for the spectral variation in local fluence is required. A straightforward method to achieve this compensation is to employ a skin model and an independent optical measurement so that the optical properties in 
skin can be measured and, thus, the spatial and spectral variation of local fluence can be estimated. However, an independent optical measurement requires simultaneous implementation of two different experimental techniques, and is technically challenging and has not yet been reported. Moreover, accuracy of a pure optical measurement of bulk tissue optical properties is still questionable [9].

Several methods have been developed to measure $\mathrm{sO}_{2}$ in both in vitro and in vivo samples based on PA measurements only. In [10] and [11], the $\mathrm{sO}_{2}$ in blood samples in a cuvette-type phantom is quantified by fitting the exponential rise in the detected PA signals. The advantage of this approach is that it is self-calibrated because it deals with the relative change in the amplitude of PA signals rather than the absolute values. However, this method relies on a large ultrasonic bandwidth, and requires that the surrounding tissues have a simple geometry and is optically homogeneous. Thus, it has limited applicability to in vivo imaging. To measure $\mathrm{sO}_{2}$ in vivo in small vessels with band-limited ultrasonic transducers, one has to develop a method to extract quantitative functional information from other features of PA signals.

In [12] and [13], in vivo two-dimensional mapping of $\mathrm{sO}_{2}$ in both brain cortical vessels and subcutaneous vessels have been reported based on the peak values of the detected PA signals which can be unambiguously attributed to different internal structures and are separable in the time domain. The smallest diameter of the imaged vessels is less then $50 \mu \mathrm{m}$. The advantage of this method is that band-limited ultrasonic transducers can be employed given that the ultrasonic center frequency satisfies the requirement of optical penetration depth in blood [14]. In [12] and 
[13], invasive methods have been employed to measure the wavelength-dependent optical attenuation in skin. This invasive method can be either measuring light transmission through an exercised skin piece or measuring PA spectrum of a subcutaneously inserted light absorbing target in vivo. The assumptions of these two methods were that the skin layer is homogeneous and the vessels locate within a short depth range from the skin surface. However, a noninvasive technique is required in clinical applications, which makes these methods impractical.

Although the wavelength-dependent fluence attenuation in skin can be roughly compensated for using above invasive measurements, such measurements can only performed at limited locations while skin optical properties can vary from depending on location and physiological state. Hence the final measured $\mathrm{sO}_{2}$ values can still be inaccurate depending on the spectral compensation factors involved. However, if the interest is in the difference of $\mathrm{sO}_{2}$ between vessels, rather than the absolute of $\mathrm{sO}_{2}$ in each vessel, the measure can be accurate regardless of the spectral compensation. Here, we demonstrate numerically that the difference of $\mathrm{sO}_{2}$ between subcutaneous vessels is minimally affected by the wavelength dependent optical attenuation in skin and present in vivo PAM experimental results which confirms our findings.

\section{Experimental setup}

The PAM system used to acquire in vivo data has been described in details previously [6,7]. Molar extinction coefficients of $\mathrm{HbO}_{2}$ and $\mathrm{HbR}$ were taken from literatures $[15,16]$. The effect of transducer focal position on the $\mathrm{sO}_{2}$ measurements was found to be relatively small [14]. 
In in vitro studies, the PA peak amplitude has been demonstrated a linear dependence with the optical absorption coefficient $\left(\mu_{a}\right)$ within the realm of $\mu_{a} \Lambda<1$, where $\Lambda$ is central frequency of the ultrasonic transducer [14]. In phantoms made from bovine blood filled cylindrical tubes, $\mathrm{sO}_{2}$ has been quantitatively measured with a high accuracy and sensitivity [13].

In this study, in vivo experiments were were carried out in Sprague Dawley rats ( 200 g, Charles River Breeding Laboratories, MA). The laboratory animal protocol for this work was approved by the Animal Studies Committee of Washington University at Saint Louis and all experimental animal procedures were carried out in conformity with the guidelines of the National Institutes of Health [17]. Change of systemic physiological states were involved in the experiments and were achieved by varying the oxygenation concentration of the inhalation anesthetic gas mixture as described in [13].

\section{Model}

The peak voltage $A$ of the PA signal generated from a blood vessel at an optical wavelength $\lambda$ is determined by the local optical fluence and several blood properties $\left(p_{\alpha}\right)$. Such properties include blood oxigenation $\mathrm{sO}_{2}=C_{\mathrm{HbO}_{2}} /\left(C_{\mathrm{HbR}}+C_{\mathrm{HbO}_{2}}\right)$ and $C_{\mathrm{Hb}}=C_{\mathrm{HbR}_{\mathrm{R}}}+C_{\mathrm{HbO}_{2}}$, where $C_{H b}$ is the total hemoglobin concentration in blood; $C_{\mathrm{HbO}_{2}}$ and $C_{\mathrm{HbR}}$ are the concentrations of $\mathrm{HbO}_{2}$ and $\mathrm{HbR}$ in blood vessel, respectively. If $A$ can be theoretically calculated from known $p_{\alpha}$ at $\lambda_{k}$, the determination of $\mathrm{sO}_{2}$ from the measured $\mathrm{PA}$ spectrum becomes a standard maximum likelihood parameter estimate problem. In fact, $A$ also depends on other parameters 
such as vessel geometry, ultrasonic bandwidth, etc. However, it has been shown that within the spectral range of $550 \mathrm{~nm}$ and $650 \mathrm{~nm}, A$ can be approximately considered a function of only the local optical fluence, $\mathrm{sO}_{2}$, and $C_{H b}$ for the purpose of $\mathrm{sO}_{2}$ measurements [14].

Unfortunately, optical fluence can only be measured at the skin surface. Inside skin, the fluence has a depth dependent distribution due to optical scattering and light absorption by melanin in epidermis and hemoglobin in capillary network in epidermis and dermis. The fluence distribution is wavelength dependent and changes with anatomical locations and physiological states. To relate optical fluence on skin surface to local fluence on vessel a skin model should be employed, which adds more unknown parameters for skin properties to the multiple parameter inversion depending on the complexity of the skin model.

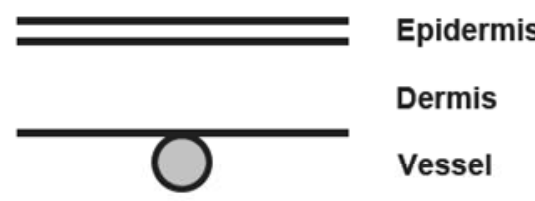

Figure 1. Schematic diagram of the skin model used in this work

In this work, a simplified skin model as shown in Figure $1[18,19]$, which contains only two layers: epidermis and dermis, is used. Each layer is treated as microscopically homogeneous layer and is described by its layer thickness and effective optical attenuation coefficient $\mu_{\text {eff }}=\sqrt{3 \mu_{a}\left(\mu_{s}^{\prime}+\mu_{a}\right)}$. The optical fluence of an incident collimated light beam is considered to attenuate exponentially after light propagating through the skin and can be expressed as 


$$
F(\lambda)=F_{0} \exp \left(-z_{e} \mu_{e f f}^{e}(\lambda)-z_{d} \mu_{e f f}^{d}(\lambda)\right)
$$

where $F_{0}$ is the optical fluence on the skin surface; $z_{e}$ and $z_{d}$ are the layer thickness of the epidermis and dermis, respectively; and $\mu_{\text {eff }}^{e}$ and $\mu_{\text {eff }}^{d}$ are the effective optical attenuation in epidermis and dermis, respectively. Although crude, this model gives reasonable approximation of optical fluence at depths larger than a few hundred micrometers [11].

The reduced optical scattering coefficients in both layers are assumed identical, which is expressed as $\mu_{s}^{\prime}=1.1 \cdot 10^{12} \lambda^{-4}+73.7 \cdot \lambda^{-0.22}[20]$. The optical absorption coefficient in epidermal layer $\mu_{a}^{e}$ is expressed as $\mu_{a}^{e}=C_{m} 6.6 \times 10^{11} \lambda^{-3.33}+\left(1-C_{m}\right) 7.84 \times 10^{7} \lambda^{-3.255}$, where $C_{m}$ is the volume fraction of melanosome, which varies from $1.3 \%$ to $43 \%$ depending on skin color [15]. In our calculation, we used $5 \%$ as Sprague Dawley rats have low melanosome concentration. Here, wavelength is in $\mathrm{nm}$, length is in $\mathrm{cm}$, and optical absorption coefficient and scattering coefficient are in $\mathrm{cm}^{-1}$. The optical absorption coefficient in dermal layer is expressed as

$$
\mu_{a}^{d}=\left(1-C_{B}\right) 7.84 \times 10^{7} \lambda^{-3.255}+\mu_{B}(\lambda) \cdot C_{B},
$$

where $C_{B}$ is the mean volume fraction of blood in dermis; $\mu_{B}(\lambda)$ is the optical absorption coefficient of blood. It is defined as $\mu_{B}=\left(\left(\varepsilon_{H b O_{2}}(\lambda)-\varepsilon_{H b R}(\lambda)\right) \cdot \mathrm{sO}_{2}^{d}+\varepsilon_{H b R}(\lambda)\right) \cdot C_{H b}$, where $\varepsilon_{\mathrm{HbO}_{2}}$ and $\varepsilon_{\mathrm{HbR}}$ is the molar extinction coefficients of $\mathrm{HbO}_{2}$ and $\mathrm{HbR}$, respectively; $\mathrm{sO}_{2}^{d}$ is the spatially averaged blood oxygenation in dermis; and $C_{H b}$ is the hemoglobin concentration in blood. In human skins, the typical value of $C_{B}$ is about $0.2 \%$. As a result, the optical absorption coefficient in dermis is related to $C_{B}, \mathrm{sO}_{2}^{d}, \varepsilon_{\mathrm{HbO}_{2}}$, and $\varepsilon_{H b R}$. 
The amplitude of the PA signal generation from the vessel located at depth $z_{e}+z_{d}$ can therefore be expressed as:

$$
A(\lambda)=K C_{H b}\left(\left(\varepsilon_{H b O_{2}}(\lambda)-\varepsilon_{H b R}(\lambda)\right) \cdot \mathrm{sO}_{2}+\varepsilon_{H b R}(\lambda)\right) \cdot F_{0} \exp \left(-z_{e} \mu_{e f f}^{e}(\lambda)-z_{d} \mu_{e f f}^{d}(\lambda)\right)
$$

where $K$ is a proportional coefficient, which is related to the transducer sensitivity, ultrasound attenuation, detection geometry, and the Grüneisen coefficient [4]. From (3), it can be seen that the only relative changes in $C_{H b}$ can be calculated quantitatively because $K F_{0}$ and $C_{H b}$ are inseparable.

The parameters discussed above are estimated by minimizing the difference between the experimental PA values $\left(A_{k}^{(e)}\right.$ measured at $\left.\lambda_{k}\right)$ and the theoretical calculation $\left(A_{k}\left(p_{\alpha}\right)\right)$ in the least squares sense. That is, the "true" values of the parameters are those who minimize the dimensionless relative error $E^{2}$ :

$$
E^{2}=\frac{\sum_{k}\left(A_{k}^{(e)}-A_{k}\left(p_{\alpha}\right)\right)^{2}}{\sum_{k}\left(A_{k}^{(e)}\right)^{2}} .
$$

Let's assume that experimental measurements have wavelength-independent normally distributed additive measurement errors, $\delta A_{k}^{(e)}$. The relation of covariance of the parameters $p_{\alpha}$, $\delta p_{\alpha} / p_{\alpha}$ to the experimental errors is given by [14]:

$$
\sum_{\alpha, \beta} \frac{\delta p_{\alpha}}{p_{\alpha}}\left(\sum_{k} S_{\alpha k} S_{k \beta}\right) \frac{\delta p_{\beta}}{p_{\beta}}=\frac{\sum_{k}\left(\delta A_{k}^{(e)}\right)^{2}}{\sum_{k}\left(A_{k}^{(e)}\right)^{2}}=\frac{\eta}{n-\eta} \bar{E} F_{\eta, n-\eta}^{(1-\gamma)},
$$


where $S_{k \alpha}=\frac{\partial A_{k}}{\partial p_{\alpha}} \frac{p_{\alpha}}{\left(\sum_{k} A_{k}^{2}\right)^{1 / 2}}$ is the sensitivity of the PA amplitude at wavelength $k$ to the parameter $p_{\alpha}, \bar{E}$ is the residual value of $E$ at convergence, $F_{\eta, n-\eta}^{(1-\gamma)}$ is a constant of the order of unity which is an F-ratio distribution with $\eta, n-\eta$ degrees of freedom for a given confidence level, $\gamma$, [21], $n$ is the number of wavelengths used and $\eta$ is the number of independent parameters. Equation (5) defines an ellipsoidal surface in $p_{\alpha}$ space projection of which on $p_{\mathrm{A}}$ axes defines normalized variance bound of a single parameter $\Delta p_{\mathrm{A}} / p_{\mathrm{A}}$. The ratio of the $\Delta p_{\alpha} / p_{\alpha}$ to error $\bar{E}$ is defined by the theoretical model used to calculate the PA signal and gives a priori estimate of error propagation from error in measurement propagates on error in parameter (so called the error propagation factor or EPF). The optimal choice of optical wavelengths used in experiment is defined by condition of minimal error in $\mathrm{sO}_{2}$ within limitations of the maximum tunable range of the dye laser. Number of used wavelength should also be relatively small to keep dataacquisition time reasonably short.

EPF for $\mathrm{sO}_{2}$ in four parameters inversion $\left(\mathrm{sO}_{2}, s \mathrm{O}_{2}^{d}, \mathrm{C}_{B}\right.$ and $\left.\mathrm{F}_{0} \mathrm{KC}_{\mathrm{Hb}}\right)$ using 8 equally spaced by $3 \mathrm{~nm}$ wavelengths, and that for two parameter inversion with $s O_{2}^{d}$ and $C_{B}$ fixed at 0.8 and 0.04 using only 4 equally spaced by $6 \mathrm{~nm}$ wavelengths is shown in Fig. 1 as a function of optical wavelength. EPF minimum for four parameters inversion is about 7 while that for two parameters inversion is about one. In both cases minima occur in wavelength range of about $570 \mathrm{~nm}=590 \mathrm{~nm}$ which was chosen for in vivo experiment. 
Results and discussion In our model, the $\varepsilon_{H b O_{2}}, \varepsilon_{H b R}$, and $C_{m}$ are taken from literature values and only the $C_{B}$ and $s O_{2}^{d}$ need to be estimated. To acquire a meaningful values for $C_{B}$ and $s O_{2}^{d}$, we fit (1) against experimental data for spectral dependence of fluence decay in rat skin. Experimental data were acquired using an invasive PA measurement. A $2 \mathrm{~mm} \times 3 \mathrm{~mm}$ rectangular piece of a $25 \mu \mathrm{m}$ thick black polyethylene film was inserted under the rat skin beneath the dermis as described in [14]. By fitting the PA spectrum from the black film insert with (4), we found out that this simplified skin model described the behaviors of the skin under different optical wavelengths fairly well. However, even for very simple case of black-insert both residual error (error in model are bias) and standard deviation (random error) are about 5\%. Taken into account that EPF for $\mathrm{sO}_{2}$ in four parameter inversion is bigger then seven, expected error in $\mathrm{sO}_{2}$ inversion can exceed $35 \%$ which makes simultaneous inversion for all vessel and tissue parameters unfeasible.

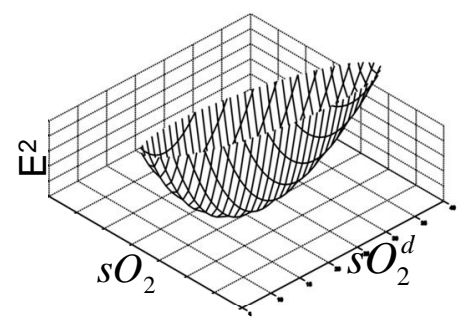

(a)

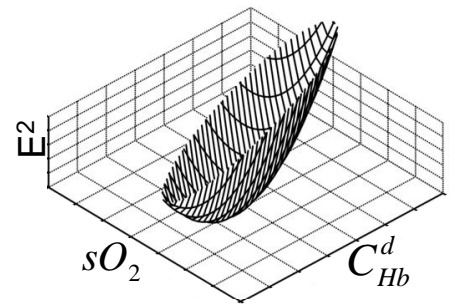

(b)

Figure 2. Error propagation factors for inversion based on different values of (a) $s O_{2}^{d}$ and (b) $C_{H b}^{d}$.

Since noninvasive absolute value of $\mathrm{s}_{2}$ in vivo may not be measurable with reasonable accuracy, it might be possible to find physiologically meaningful combination of $\mathrm{sO}_{2}$ related values which are less dependent on skin optical properties and can be accurately measured in vivo. First, we 
estimated the influence of $s O_{2}^{d}$, and $C_{B}$ ranging from 0 to 1 (all possible values) and 0 to $1.2 \%$ (up to more than twice of the maximum measured value), respectively on $\mathrm{sO}_{2}$ inversion. For $s \mathrm{O}_{2}$ ranging from $30 \%$ to $100 \%$ we calculate $A_{k}\left(p_{\alpha}\right)$ at optical wavelengths $578,584,590$, and $596 \mathrm{~nm}$ according to (4), treat them in as an error free experimental data and plot the result of two parameter $\left(\mathrm{F}_{0} \mathrm{KC}_{\mathrm{Hb}}\right.$ and $\mathrm{sO}_{2}$ ) inversion for $\mathrm{sO}_{2}$ completely ignoring skin light attenuation in Figure 2. As one can see, skin optical attenuation defined by $s O_{2}^{d}$, and $C_{B}$ dramatically affect inverted $\mathrm{sO}_{2}$ value. However, relationship of the inverted $\mathrm{sO}_{2}$ values to the exact $\mathrm{sO}_{2}$ values remains relatively linear. Moreover, slope of the curves in Fig. 3a does not change with $s O_{2}^{d}$, and $\mathrm{C}_{B}$ as much as $\mathrm{sO}_{2}$ percentage value. It indicates that within reasonable bounds, at least relative changes in $\mathrm{sO}_{2}$ can be measured without preliminary knowledge of skin optical properties albeit with some bias exist as seen from the change of slope of curves in Figure 2a. Taking a reasonable suggestion that $\mathrm{SO}_{2}$ in arterial blood under hyperoxia is $\sim 100 \%$, bias of two parameter $\mathrm{sO}_{2}$ inversion can thus be decreased to less than $\pm 1 \%$ using simple linear empirical formula, coefficients of which can depend on chosen optical wavelength range and vessel depth:

$$
s \mathrm{O}_{2}^{(e m)}=s \mathrm{O}_{2} \cdot\left(1+0.82 s \mathrm{O}_{2}(100 \%)\right) / 1.863+1-s \mathrm{O}_{2}(100 \%) .
$$

Above analysis suggests that the relative changes in $\mathrm{sO}_{2}$ in vessels are minimally affected by $s \mathrm{O}_{2}^{d}$ and $C_{B}$. Such relative changes could be, for example, different in $\mathrm{sO}_{2}$ between two neighboring vessels. 


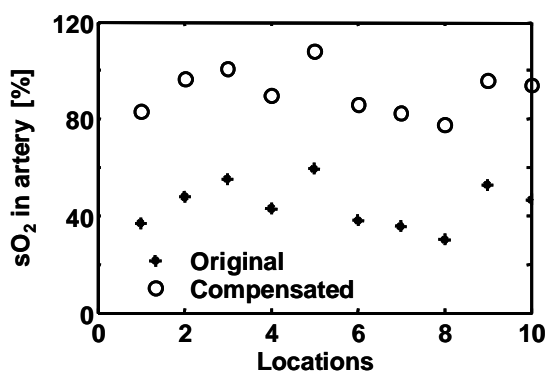

(a)

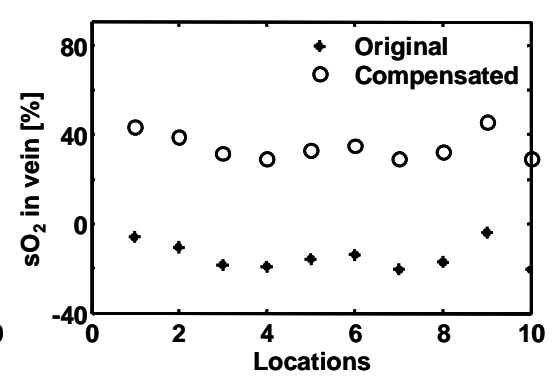

(b)

Figure 3. Calculated $\mathrm{sO}_{2}$ values in arteries (a) and veins (b) before and after spectral compensation.

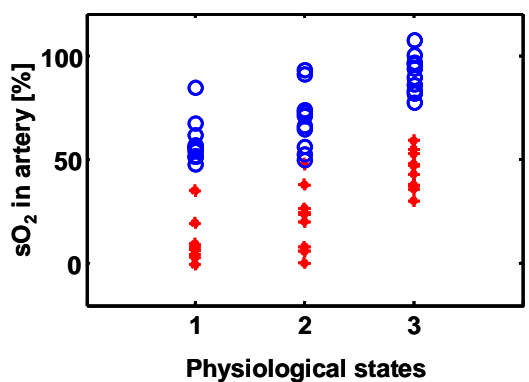

(a)

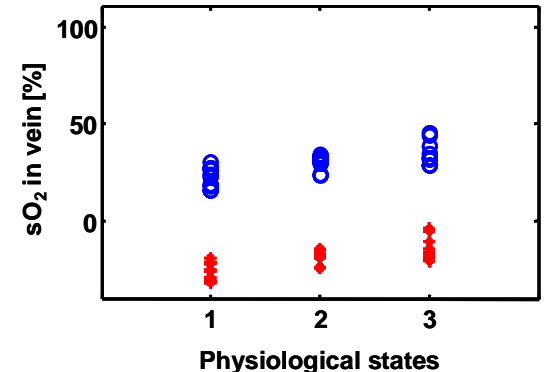

(b)

Figure 4. $\mathrm{sO}_{2}$ values in arteries (a) and veins (b) before and after spectral compensation in three different systemic physiological states. 1: hypoxia, 2: normoxia; and 3: hyperoxia.

To further verify this conclusion, we compared $\mathrm{sO}_{2}$ differences between a typical artery and vein at multiple measurement locations before and after spectral compensation of the spectral fluence variation at three physiological states in vivo. The calculation of $\mathrm{sO}_{2}$ followed the description in $[13,14]$ and the inverse of the PA spectrum of the black film was used as the spectral compensation factor at corresponding optical wavelengths. As previously reported, arteries and veins can be separated based on the imaged $\mathrm{sO}_{2}$, we selected one artery and one vein to be our target vessels in this work. Ten measurement locations were then selected along each selected 
vessel. At each measurement location, $\mathrm{sO}_{2}$ values before and after spectral compensation were calculated in both vessels. As shown in Figure $3 a$ and Figure $3 b$, the two sets of inverse calculations are parallel to each other in vein and artery, respectively, though the values after spectral compensation are much more realistic. Based on the results shown in Figure 3, the calculated differences in $\mathrm{sO}_{2}\left(\Delta \mathrm{sO}_{2}\right)$ between vein and artery for the 10 measurement show little dependence on spectral compensation as well. The variation is $\Delta \mathrm{sO}_{2}$ before and after spectral compensation is $2.42 \% \pm 0.58 \%$. Hence, it can be expected that even without a reasonable spectral compensation, a reliable estimation of variation of $\mathrm{sO}_{2}$ can be experimental achieved. Furthermore, $\Delta \mathrm{sO}_{2}$ was shown not to change much under all the three physiological states as shown in Figure 4. This observation demonstrates that, functional parameters such as local oxygen consumption can be quantified based on only PA measurement noninvasively. Such capability shows potential applications in, for example, tumor physiology study where consumptions of oxygen by tumor cells at various developing stages can be measured; and functional brain imaging, where different levels of neural reactions to external stimulations is related to the oxygen consumptions.

\section{References}

[1] C.G.A. Hoelen, et al., "Three-dimensional photoacoustic imaging of blood vessels in tissue," Opt. Lett. 23 648-650 (1998).

[2] A.A.Oraevsky and A.A. Karabutov, Optoacoustic Tomography. in Biomedical Photonics Handbook, Vol. PM125 (ed. Vo-Dinh, T.) (CRC Press, Boca Raton, Florida 2003). 
[3] R.A. Kruger, et al., "Photoacoustic ultrasound (PAUS) - reconstruction tomography," Medical Phys. 22 1605-1609 (1995)

[4] M. Xu, et al., "Photoacoustic imaging in biomedicine," Rev. Sci. Instrum. 77041101 (2006).

[5] X. Wang, et al., "Noninvasive laser-induced photoacoustic tomography for structural and functional imaging of the brain in vivo," Nat. Biotech. 21 803-806 (2003).

[6] K. Maslov, et al., "In vivo dark-field reflection-mode photoacoustic microscopy," Opt. Lett. 30 625-627 (2005).

[7] H. F. Zhang, et al., "Functional photoacoustic microscopy for high-resolution and noninvasive in vivo imaging," Nat. Biotech. 24 848-851 (2006).

[8] H. F. Zhang, et al., "In vivo volumetric imaging of subcutaneous microvasculature by photoacoustic microscopy," Opt. Express 14 9317-9323 (2006).

[9] G. Greisen "Is near-infrared spectroscopy living up to its promises?" Seminars in Fetal \& Neonatal Medicine (2006), doi:10.1016/j.siny.2006.07.010.

[10] R. O. Esenaliev, et al., "Optoacoustic technique for noninvasive monitoring of blood oxygenation: a feasibility study," Appl. Opt. 41 4722-4731 (2002).

[11] J. Laufer, et al., "In vitro measurements of absolute blood oxygen saturation using pulsed near-infrared photoacoustic spectroscopy: accuracy and resolution,” Phys. Med. Bio.l 50 4409-4428 (2004).

[12] X. Wang, et al., "Non-invasive imaging of hemoglobin concentration and oxygenation in the rat brain using high-resolution photoacoustic tomography," J. Biomed. Opt. 11024015 (2006).

[13] H. F. Zhang, et al., "Imaging of hemoglobin oxygen saturation variations in single vessels in vivo using photoacoustic microscopy,” Appl. Phys. Lett. 90053901 (2007).

[14] M. Sivaramakrishnan, et al., "Limitations of quantitative photoacoustic measurements of blood oxygenation in small vessels," Phys. Med. Biol. 52 1349-1361 (2007).

[15] S. L. Jacques and S. A. Prahl 2004 Absorption spectra for biological tissues http://omlc.ogi.edu/spectra/hemoglobin/index.html Oregon Medical Laser Center OR 
[16] W. G. Zijlstra, et al., Visible and near infrared absorption spectra of human and animal hemoglobin, determination and application (VSP The Netherlands, 2000)

[17] National Institute of Health Publication No. 86-23 1985 U.S. GPO Washington DC

[18] M. J. C. Van Gemert, et al., "Skin optics," IEEE Trans. Biomed. Eng. 36 1146-1154 (1989).

[19] S. L. Jacques 1989 Skin optics http://omlc.ogi.edu/news/jan98/skinoptics.html Oregon Medical Laser Center OR

[20] A. N. Bashkatov1, et al., "Optical properties of human skin, subcutaneous and mucous tissues in the wavelength range from 400 to 2000nm," J. Phys. D: Appl. Phys. 38 2543-2555 (2005).

[21] Lewicki P, et al., Statistics methods and applications, (StatSoft Inc, Tulsa, OK, 2006) 